\title{
Distributed Storage Management Using Dynamic Pricing in a Self-Organized Energy Community
}

\author{
Ebisa Negeri and Nico Baken \\ Delft University of Technology, Delft, The Netherlands \\ \{E.O.Negeri, N.H.G.Baken\}@tudelft.nl
}

\begin{abstract}
We consider a future self-organized energy community that is composed of "prosumer" households that can autonomously generate, store, import and export power, and also selfishly strive to minimize their cost by adjusting their load profiles using the flexibly of their distributed storage. In such scenario, the aggregate load profile of the selforganized community is likely to be volatile due to the flexibility of the uncoordinated selfish households and the intermittence of the distributed generations. Previously, either centralized solutions or cooperation based decentralized solutions were proposed to manage the aggregate load, or the load of an individual selfish household was considered. We study the interplay between selfish households and community behavior by proposing a novel dynamic pricing model that provides an optimal price vector to the households to flatten the overall community load profile. Our dynamic pricing model intelligently adapts to the intermittence of the DGs and the closed-loop feedback that might result from price-responsiveness of the selfish households using its learning mechanism. Our dynamic pricing scheme has distinct import and export tariff components. Based on our dynamic pricing model, we propose a polynomial-time distributed DS scheduling algorithm that runs at each household to solve a cost minimization problem that complies with the selfish nature of the households. Our simulation results reveal that our distributed algorithm achieves up to $72.5 \%$ reduction in standard deviation of the overall net demand of the community compared to a distributed scheduling with two-level pricing scheme, and also gives comparable performance with a reference centralized scheduling algorithm.
\end{abstract}

\section{Introduction}

With the growing concerns for sustainable energy, an increasing number of diverse types of small-scale distributed generations (DGs), such as solar panels, are penetrating into neighborhoods. As a consequence, households are evolving from passive consumers towards active prosumers (producer-consumer) that can autonomously generate, store, export or import power. According to the EU parliament, all buildings built after 2019 will have to produce their own 
energy on site1. As households take power into their own hands, they tend to self-organize and form an "energy community" [1], for example at neighborhood level, to locally exchange power with each other and to influence the market with "the power of the collective." In such an autonomous community, the aggregate community load profile is likely to be highly volatile mainly because of two reasons. Firstly, the production of the DGs is highly intermittent. Secondly, the households could use the flexibility offered by their distributed storages (DSs), through charging and discharging, to selfishly adjust their load profile to minimize their cost, which could add up to a volatile aggregate profile such as large peaks that result if many households charge their DS at the same time. Indeed, DSs are becoming more attractive at the household level, especially along with intermittent DG, because they can increase demand-side flexibility and decrease the consumers' costs for electricity supply [2]. The volatility has various disadvantages. Firstly, a large number of high cost and carbon intensive "peak-plants" are required to provide power alongside the intermittent sources to compensate the variability. Moreover, the power peaks resulting from the variability may lead to infrastructure damages and expensive capacity upgrading. Thus, leveling the volatile overall community profile is crucial.

There are a few works in the literature on scheduling a system of multiple storage units ([3]-4]). In these works, however, either the storage units are centrally scheduled without obeying the autonomy of the households, or households cooperatively play a game in a decentralized way with similar learning behavior to achieve a desired communal profile. Recently, dynamic pricing based demandresponse strategy is gaining attention as a coordination mechanism. When the households are very flexible (for instance, by using their DSs), their responsiveness to the dynamic price might lead to a closed-loop feedback that could cause volatility, as demonstrated in 5. Thus, in addition to reflecting the aggregate load profile, a dynamic pricing model needs intelligence to capture the flexibility of the households to respond to price signals.

In this paper, we consider a self-organized neighborhood energy community that is composed of selfish prosumer households, where power can be exchanged horizontally between the households within the community, and bidirectionally between the autonomous energy community and the rest of the grid. The energy community is controlled using a multi-agent based electronic market proposed in 6], where the households can trade with each other using local tariffs. A household-agent coordinates the resources at the household to optimize the household consumption, while a community-agent watches over the overall load profile of the energy community and coordinates the household-agents accordingly. The community is assumed to have smart grid capabilities [7, such as intelligent devices at each household that can exchange information across a communications platform.

The main contributions of this paper are:

${ }^{1}$ European Parliament, "All New Buildings to be Zero Energy from 2019," Committee on Industry, Research and Energy, Brussels 2009. 
- We study coordination in a future energy community that is formed by a group of self-organized selfish prosumer households that can generate, store, import and export power, whereby the aggregate load profile is likely to be highly volatile due to the flexibility and selfish behavior of the prosumer households as well as the intermittence of DGs.

- We propose a dynamic pricing model that embodies a learning mechanism to iteratively adapt to the responsiveness of the households in the self-organized energy community and the intermittence of the DGs. Our pricing model has distinct import and export tariffs that handle the power import and export of the prosumers.

- We propose a polynomial-time distributed DS scheduling algorithm that runs at each household to solve a cost minimization problem of the selfish household, and at the same time flattening the overall community load profile using our dynamic pricing model.

- Our simulation results reveal that our distributed scheduling algorithm gains comparative performance with a reference centralized scheduling algorithm, and also achieves up to $72.5 \%$ reduction in standard deviation of the overall net demand of the community compared to a two-level pricing scheme.

The rest of the paper is structured as follows. Section 2 presents the mathematical model of the DS scheduling problem. In Section 3 the dynamic pricing model is presented, followed by our distributed algorithm in Section 4 . The simulation results are presented in Section 5 Finally, our concluding remarks are presented in Section 6.

\section{System Model}

We present a formulation of the problem of finding an optimal schedule for DSs to flatten the demand profile of a self-organized neighborhood energy community. The distributed storage systems are scheduled offline over a scheduling time period $\tau(24 \mathrm{~h})$ that is divided into time steps, i.e. $\tau=\{1,2, \ldots, T\}$, that have equal duration of $\Delta$ time units.

\subsection{Distributed Storage (DS)}

Each DS is battery storage and is described by the parameters that are summarized below 9 .

$\Phi$ : the maximum energy storage capacity (in $\mathrm{kWh}$ ).

$\alpha$ : the maximum power capacity of the rectifier (in $\mathrm{kW}$ ).

$\beta$ : the maximum power capacity of the inverter (in $\mathrm{kW}$ ).

$\nu$ : the time required for a full charge cycle (in h).

$\eta^{s t}$ : the cycle efficiency (\%).

$\eta^{r e c}$ : the rectifier efficiency (\%).

$\eta^{i n v}$ : the inverter efficiency (\%).

$\delta$ : maximum depth of discharge allowed (\%). 
We neglect the self-dissipation of the battery, because it has a negligible effect for the length of our scheduling period. In addition to the above parameters, the variables $X_{j}$ and $Y_{j}$ are used to denote the amount of power that is charged into and discharged from, respectively, the DS in time step $j$. Assuming a symmetric cycle efficiency, $\eta^{s t}$, we denote the storage charging efficiency and discharging efficiency by $\eta^{c}$ and $\eta^{d}$, respectively, where $\eta^{c}=\eta^{d}=\sqrt{\eta^{s t}}$. The state of charge (SOC) of the DS at time step $j$ is denoted by $\Psi_{j}$ (in $\mathrm{kWh}$ ).

Generally, the SOC of a DS at the end of a time step $k$ can be obtained from the SOC at the end of the previous time step $j$ as shown in Eq. 1. The term added to $\Psi_{j}$ accounts for the net rise in the storage level in time step $k$ which is the difference between the stored energy due to charging of the storage and the discharged energy from the DS. The number of time units per time step $(\Delta)$ is used in Eq. 1 to convert power $(\mathrm{kW})$ into energy $(\mathrm{kWh})$. The maximum SOC of each DS is bounded by its maximum storage capacity (Eq. 2). The SOC cannot fall below the maximal discharging depth $\delta$ (Eq. 3). The rate of charging a DS is limited by the maximum charging rate $\left(\frac{\Phi}{\nu}\right)$ and the maximum power capacity of the rectifier $\alpha$ (Eq. 4), whereas the discharging rate of the DS is limited by the maximum power capacity of the inverter $\beta$ (Eq. 51).

$$
\begin{aligned}
\Psi_{k} & =\Psi_{j}+\left(\Delta \eta^{r e c} \eta^{c} X_{k}-\Delta \frac{Y_{k}}{\eta^{i n v} \eta^{d}}\right) \\
\Psi_{j} & \leq \Phi, \forall j \in \tau \\
\Psi_{j} & \geq(1-\delta) \times \Phi, \forall j \in \tau \\
X_{j} & \leq \min \left(\alpha, \frac{\Phi}{\nu}\right), \forall j \in \tau \\
Y_{j} & \leq \beta, \forall j \in \tau
\end{aligned}
$$

\subsection{Demand and Production}

The accumulated power demand of all the appliances of a given household in time step $j$ is denoted by $D_{j}$, whereas we designate the total power production of all the distributed sources owned by the household in time step $j$ by $P_{j}$. In this work, we assume that forecasts of $D$ and $P$ are available for the entire scheduling period. We define the scheduled demand of a household in time step $j$, denoted by $R_{j}$, as the difference between the demand and the self supply of the household in the time step (Eq. 6). The amount of power the household imports from or exports to the grid cannot exceed the power capacity of the cable connecting the household to the grid, denoted by $\omega$, as shown in Eq. 7 . The scheduled demand of the overall neighborhood $R_{j}^{o}$ in time step $j$ is given by the sum of the scheduled demands of all the households in the neighborhood as shown in Eq. 8. We represent the net demand of the community ignoring the DSs by $Q_{j}^{o}$, for each time step $j$ (Eq. 9).

$$
\begin{aligned}
& R_{j}=\left(D_{j}+X_{j}\right)-\left(Y_{j}+P_{j}\right), \forall j \in \tau \\
& R_{j} \leq \omega, \forall j \in \tau
\end{aligned}
$$




$$
\begin{aligned}
& R_{j}^{o}=\sum_{i} R_{j}, \forall i \text { households } \\
& Q_{j}^{o}=\sum_{i}\left(D_{j}-P_{j}\right), \forall i \text { households }
\end{aligned}
$$

\subsection{The Cost}

Since a household can import power from the grid as well as feed the surplus power into the grid, we will have two different tariffs. We denote the import tariff and the export tariff in time step $j$ by $\theta_{j}$ and $\lambda_{j}$, respectively. The electricity cost of a household in time step $j$ is denoted by $C_{j}$, and is given in Eq. 10. Clearly, $C_{j}$ is a positive cost when power is imported $\left(Q_{j}>0\right)$, and is a negative cost (income) when power is exported $\left(Q_{j}<0\right)$.

$$
C_{j}=\left\{\begin{array}{l}
\theta_{j} \times Q_{j}, \text { if } Q_{j} \geq 0 \\
\lambda_{j} \times Q_{j}, \text { if } Q_{j}<0
\end{array}, \forall j \in \tau\right.
$$

\subsection{Optimization Problems}

Given a self-organized neighborhood energy community of $N$ prosumer households, each with forecasted values of demands and self productions and owning a DS with known capacities and efficiency parameters, the problem of optimally scheduling the $N$ DSs over a scheduling period $\tau$ to achieve a flattened overall community load profile is addressed differently by the centralized and distributed scheduling strategies.

The centralized scheduling strategy gives the community-agent the authority to centrally schedule all the DSs of the households in the community. The community-agent first computes the average value of the net demand of the community (Eq. 11), and then schedules the DSs trying to minimize the deviation of the overall scheduled demand of the community from the average value. The corresponding quadratic optimization problem is formulated in Eq. 12. where $\mathbf{X}_{1, . ., N}$, and $\mathbf{Y}_{1, . ., N}$ represent the charging and discharging schedules, respectively, of the $N$ DSs. Although this approach does not comply with the autonomy and selfish nature of the households, we use it as a reference.

$$
\text { mean }=\frac{1}{T} \sum_{j=1}^{T} Q_{j}^{o}
$$

$$
\underset{X_{1} \ldots X_{N}}{\operatorname{minimize}} \sum_{j \in \tau}\left(R_{j}^{o}-\text { mean }\right)^{2}
$$

\section{subject to:}

Equations 1, 9 
In the distributed scheduling technique each household-agent autonomously schedules its DS to selfishly minimize the cost of that household. The communityagent coordinates the household-agents by providing a novel dynamic pricing based incentive to achieve a flattened overall profile of the self-organized energy community. While the linear programming formulation of the household cost optimization problem is given in Eq. 13, the dynamic pricing model and the details of the distributed algorithm are deferred to Sections 3 and 4, respectively.

$$
\underset{X, Y}{\operatorname{minimize}} \sum_{j \in \tau} C_{j}
$$

subject to: Equations 1, 7

\section{The Dynamic Pricing Model}

Our dynamic pricing model is based on a scenario where the autonomous households trade electricity with each other on a local electronic energy market 6] in the community whereby the autonomy of the energy community allows the community-agent to propose local price-vectors to achieve desirable communal goals. In our dynamic pricing model, the community-agent proposes a price vector, then each household-agent selfishly schedules the storage unit of that household by solving Eq. 13 and replies with the corresponding scheduled demand of the household. Based on the response of all the households, the communityagent adjusts the price vector using an intelligent learning mechanism. This is repeated iteratively to obtain an optimal price vector that yields a flattened overall community profile.

In the proposed pricing model, the cost of a unit power varies from one time step to another. The optimal tariff for each time step is determined iteratively. At the $(k+1)^{t h}$ iteration cycle, the tariff for the $j^{\text {th }}$ time step is obtained by adjusting the corresponding tariff in the previous iteration cycle $k$ using an incremental factor $\left(\gamma \times \xi_{k, j}\right)$ :

$$
\theta_{k+1, j}=\theta_{k, j}\left(1+\left(\gamma \times \xi_{k, j}\right)\right)
$$

The incremental factor is composed of two terms: the learning factor $(\gamma)$ and the deviation factor $\left(\xi_{k, j}\right)$. The learning factor is a constant $(0<\gamma \leq 1)$ that determines to which extent the deviation factor overrides the old price vector. Empirical optimal values of $\gamma$ are provided in Section 5 . The deviation factor captures the variation of $R^{o}$ from its average value. Let $R_{k, j}^{o}$ be the overall scheduled demand of the neighborhood in the $j^{t h}$ time step for the $k^{\text {th }}$ iteration cycle. Let mean* be the average of the overall scheduled demand (Eq. 15), then the deviation factor, $\xi_{k, j}$, is given in Eq. 16, where sign determines whether the incremental value should be positive or negative. If $Q_{k, j}^{o}>$ mean $^{*}$, then we add a positive incremental value to the tariff to reduce consumption in this time step: sign $=1$. If $Q_{k, j}^{o}<$ mean $^{*}$, then we subtract an incremental value from the tariff to increase consumption in this time step: sign $=-1$. 


$$
\begin{aligned}
\text { mean }^{*} & =\frac{1}{T} \sum_{i=1}^{T} R_{k, j}^{o} \\
\xi_{k, j} & =\operatorname{sign} \times \frac{\left(R_{k, j}^{o}-\text { mean }^{*}\right)^{2}}{\sum_{i=1}^{T}\left(R_{k, i}^{o}-\text { mean }^{*}\right)^{2}}
\end{aligned}
$$

$\xi_{k, j}$ reflects the effect of the offset of the scheduled demand from the mean value on the price vector. The pricing model increases the tariff on the time steps where the overall scheduled demand of the neighborhood $\left(R^{o}\right)$ is above the average, and reduces the tariff when $R^{o}$ is below the average, thereby providing incentives to the selfish households to flatten the overall scheduled neighborhood demand. Since the price vector is improved iteratively depending on the reaction of the households, the intelligent learning employed by our dynamic pricing model effectively handles the price-responsiveness of the households, thereby minimizing the closed-loop feedback effect. In effect, the aggregate load profile of the self-organized energy community could be managed.

Following the suggestion in [8], the power feed-in tariffs are obtained by subtracting the transport part of the import tariffs (Eq. 17), where $\theta^{t r}$ is the transport part of the import tariff $\theta_{j}$. The pricing model can be modified to achieve objectives other than flattening the overall profile by simply substituting the mean $^{*}$ in Eq. 16 by the desired target value for each time step.

$$
\lambda_{j}=\theta_{j}-\theta^{t r}, \forall j \in \tau
$$

\section{The Distributed Algorithm}

Our design of the distributed algorithm reflects the autonomy and selfishness of the households, while simultaneously striving to flatten the aggregate load profile of the self-organized energy community using our novel dynamic pricing model. The block diagram describing the execution of our distributed scheduling algorithms is shown in Fig. 1. The algorithm embodies a fixed number of iterative cycles that search for an optimal price vector that yields the best level of flatness for the overall scheduled net demand of the energy community $\left(R^{o}\right)$. We describe the flatness of $R^{o}$ in terms of its standard deviation:

$$
\sigma=\sqrt{\frac{1}{T} \sum_{j=1}^{T}\left(R_{j}^{o}-\text { mean }^{*}\right)^{2}}
$$

The algorithm is initiated at the community-agent. At each iteration step, the community-agent sends the recent tariff vectors $(\theta, \lambda)$ to each household-agent. Upon receiving the tariff vectors, each household-agent solves its selfish cost optimization problem (Eq. 13) with the up-to-date tariffs, and sends its resulting scheduled demand to the community-agent. When the community-agent receives 
the scheduled demands of all the households in the current iteration cycle, it computes the tariff vectors for the next iteration cycle using Eq. 14, and the iteration continues. Once the desired fixed number of iterations is performed, the community-agent picks the best tariff vectors that yielded the smallest value of $\sigma$, and the corresponding schedules of the DSs are adopted.

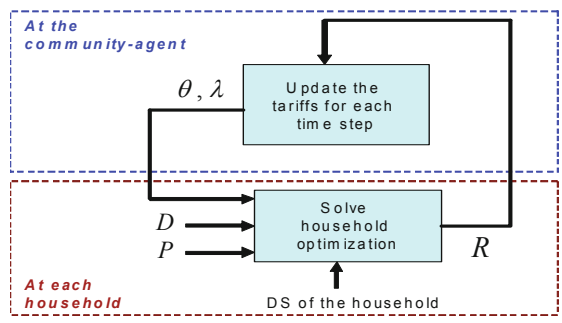

Fig. 1. A block diagram for the distributed algorithm

\section{Simulation}

In our simulation, storage systems installed at all the households have the same parameter specifications. Lead-acid battery storage is used as a reference, since it is regarded as the most economical choice 9 . The parameter specifications for lead-acid suggested in 9] are listed in Table 1. The data for the demand and electricity production of a household are acquired from Alliander 2 . The distributed sources of a household are a PV panel and a micro-CHP. The data were obtained by field measurements that were taken every 15 minutes for a duration of 24 hours using smart meters installed at the households. The microCHP generation is constant $(1 \mathrm{~kW})$ over each period of operation, and the data specifies the start time and end time of each period of its operation. To make alternative profiles for each household, we randomized the values according to a normal distribution. For demand and PV panel production, the randomized profiles were generated with mean value equal to the measured data and standard deviation equal to $15 \%$ of the mean, for each time step. For the micro-CHP production, different variants of the start time and end time of the production periods are generated using the normal distribution function with mean values equal to the corresponding measured values and standard deviation of one time step (15 minutes). A sample of the daily profile of a household in Spring season is shown in Fig. 2 .

The transport part of the electricity import tariff is $\theta^{t r}=0.04 / k W h$. The data is obtained from $\mathrm{ECN}^{3}$. Thus, for each time step $j$, the feed-in tariff is obtained as $\lambda_{j}=\theta_{j}-0.04 / k W h$.

\footnotetext{
${ }^{2}$ Alliander is the largest electricity distribution network operator company in the Netherlands owning $40 \%$ of the distribution networks.

${ }^{3}$ Energy Research Centre of The Netherlands, http://www.energie.nl/.
} 


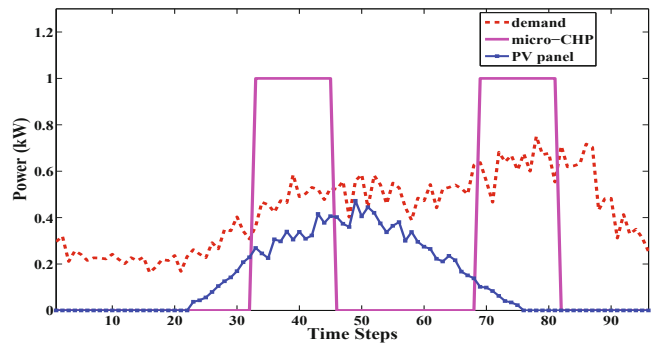

Fig. 2. Demand and production profiles of a household

Table 1. Parameter Values of the Storage System [1]

\begin{tabular}{|c|c|c|c|c|c|c|c|}
\hline$\Phi$ & $\nu$ & $\delta$ & $\eta^{s t}$ & $\alpha$ & $\beta$ & $\eta^{r e c}$ & $\eta^{i n v}$ \\
\hline $\mathrm{kWh}$ & $\mathrm{h}$ & $(\%)$ & $(\%)$ & $\mathrm{kW}$ & $\mathrm{kW}$ & $(\%)$ & $(\%)$ \\
\hline 5 & 5 & 80 & 85 & 1 & 0.5 & 95 & 98 \\
\hline
\end{tabular}

The proposed algorithm is simulated with different demand and generation data sets representing each of the four seasons of the year. In addition to the centralized scheduling and the dynamic pricing based distributed scheduling, we have a simulation scenario in which households autonomously schedule their DSs to minimize their cost responding to fixed two level tariffs (day and night tariffs).

The relative performance of the scheduling algorithms was similar under the data sets representing the four seasons, thus we present only the results obtained using the data sets representing the Spring season due to limited space. Fig. 3 shows the overall scheduled demand of the community obtained using the centralized and the distributed scheduling techniques. The large negative net demands of the neighborhood in the time steps 32-45 and 67-80 result from the accumulated production of the micro-CHPs and the PV panels that exceed the total demand. Apparently, both the centralized and distributed scheduling techniques have achieved significant improvement $(75.6 \%$ and $75.0 \%$ reductions in $\sigma$, respectively) in the overall scheduled demand profile compared to the net demand of the community. It is interesting to see that the distributed technique, that maintains the autonomy and selfishness of the households, has achieved a comparable performance with the centralized technique that makes a simplifying assumption that all the DSs are centrally managed. The achievement of the distributed technique stems from the intelligence of its dynamic pricing model based incentive.

The comparison of the performance of our dynamic pricing based distributed scheduling technique to that of the two level pricing scheme shown in Fig. 4 reveals that our distributed algorithm achieves a profile with significant improvement in flatness compared to the two level pricing. Apparently, the scheduled demand using two price levels did not show significant improvement on the shape 


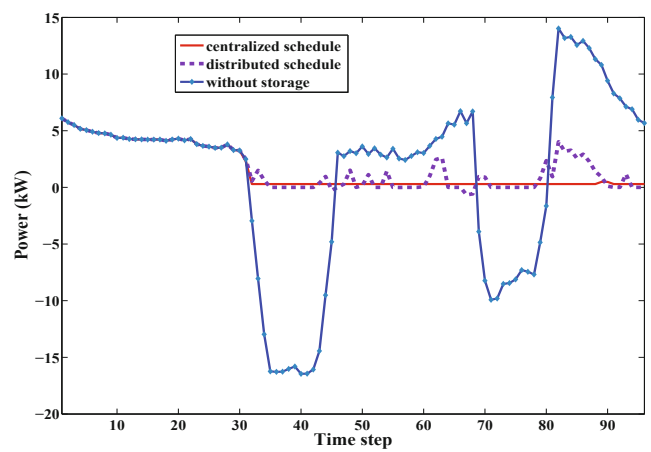

Fig. 3. Comparison of the centralized and distributed algorithms using the overall scheduled demand for $\mathrm{N}=20$

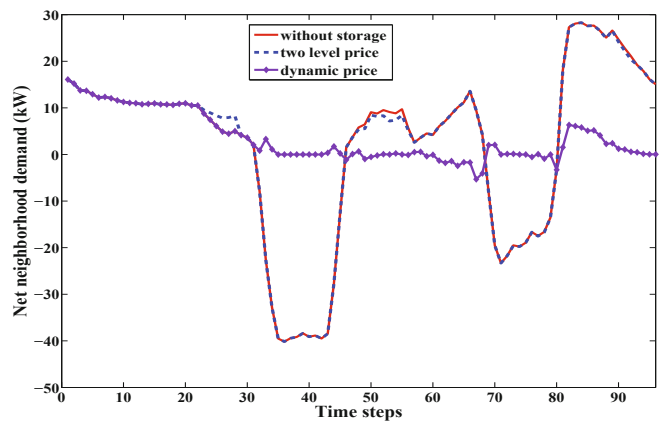

Fig. 4. Comparison of the dynamic pricing and two-level pricing schemes using the overall scheduled demand for $\mathrm{N}=50$

of the overall net demand, implying that the pricing scheme does not incentivise the households enough. Our simulation results based on the data representing the four seasons of a year reveal that our dynamic pricing based algorithm can achieve up to $72.5 \%$ improvement compared to the two-level pricing scheme in terms of the standard deviation of the overall scheduled demand.

A comparison of the performance of the distributed algorithm under different values of the learning factor $(\gamma)$ is shown in Fig. 5. The figure shows that the standard deviation of the overall scheduled demand decreases slowly for smaller values of $\gamma$, whereas, it drops rapidly for larger values of $\gamma$. On the other hand, the standard deviation curve is more stable for smaller values of $\gamma$. Furthermore, no significant improvement in $\sigma$ is observed after a fixed number of iterations. Hence, the algorithm is expected to perform well with few iterations using the larger values of $\gamma(0.2 \leq \gamma \leq 0.5)$. 


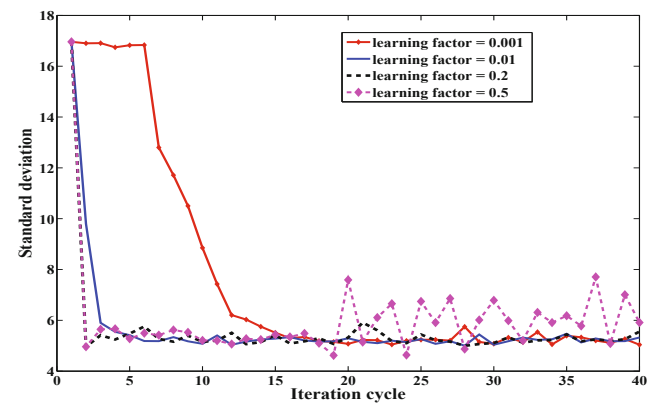

Fig. 5. The effect of the learning factor

\begin{tabular}{|c|c|c|c|c|c|c|c|}
\hline \multicolumn{8}{|c|}{$\begin{array}{r}\text { Performance deviations ( } \% \text { value) due to parameter value vari } \\
\text { Reference value variation }\end{array}$} \\
\hline Parameter & Ref. value & $-20 \%$ & $-10 \%$ & $-5 \%$ & $+5 \%$ & $+10 \%$ & $+20 \%$ \\
\hline \multirow{3}{*}{$\begin{aligned} \text { Cycle eff. } & \eta^{s t} \\
\text { Rectifier eff. } & \eta^{r e c} \\
\text { Inverter eff. } & \eta^{i n v}\end{aligned}$} & 82 & -4.6 & -2.4 & -1.3 & 1.4 & 5.2 & 6.8 \\
\hline & 95 & -7.9 & -4.9 & -0.4 & 0.4 & $\mathrm{n} / \mathrm{a}$ & $\mathrm{n} / \mathrm{a}$ \\
\hline & 98 & -9.0 & -1.2 & -0.04 & $\mathrm{n} / \mathrm{a}$ & $\mathrm{n} / \mathrm{a}$ & $\mathrm{n} / \mathrm{a}$ \\
\hline \multirow{3}{*}{$\begin{array}{l}\text { Max. charg. rate, } \min \left(\alpha, \frac{\Phi}{v}\right) \\
\text { Max. disch. rate, } \beta\end{array}$} & & $-50 \%$ & $-25 \%$ & $-10 \%$ & $+10 \%$ & $+25 \%$ & $+50 \%$ \\
\hline & 1 & -9.3 & -0.03 & -0.01 & 0.01 & 0.01 & 0.07 \\
\hline & 0.5 & -24.5 & -7.3 & -1.7 & 1.7 & 2.6 & 2.9 \\
\hline \multirow[t]{2}{*}{ Community size, $N$} & 50 & 1 & 10 & 20 & 30 & 40 & 50 \\
\hline & & -5.41 & -0.97 & -0.31 & -0.12 & -0.1 & 0 \\
\hline \multirow[t]{2}{*}{ Storage capacity, $\Phi$} & 5 & 1 & 2 & 3 & 7 & 9 & 11 \\
\hline & & 137 & 41 & 0 & 0 & 0 & 0 \\
\hline
\end{tabular}

Fig. 6. Algorithm performance in response to perturbing parameters

The reference parameter values in Table 1 are perturbed to assess their impact on the performance of our distributed algorithm. The results in Fig. 6reveal that varying the efficiency parameters, the maximum charging and discharging rates of the DS results in low variations (mostly between $-10 \%$ and $+10 \%$ ) in the performance of the algorithm, where increasing the values of the parameters increases flexibility of the households to respond to the dynamic price, thereby slightly increasing performance. Varying the community size also resulted in small performance deviation which results form the randomization of the profiles of the households. On the other hand, with very low storage capacity, the households have limited flexibility to store and release power in response to the dynamic price, thereby leading to larger values of $\sigma$.

\section{Conclusions}

The load profile of a self-organized energy community composed of selfish prosumers that can autonomously generate, store, import, and export power, is likely to be highly volatile due to the intermittence of the distributed generations and the autonomy and selfish nature of the prosumers. We have introduced 
a novel dynamic pricing model that can help to flatten the load profile in such scenario by intelligently adapting to the price-responsiveness of the selfish prosumers using its learning mechanism. We have proposed a distributed scheduling algorithm of distributed power storages that uses the dynamic pricing model to flatten the community load profile while each household selfishly tries to minimize its cost by solving a simple polynomial-time optimization problem formulated via linear programming. Simulation results reveal that our distributed algorithm has comparable performance with a reference centralized scheduling algorithm, and it can achieve up to $72.5 \%$ improvement in standard deviation of the load profile of the community compared to a reference two-level pricing model.

Our distributed algorithm and dynamic pricing model can also be extended to schedule flexible electrical units such as electric vehicles, heat-pumps, etc., and could be easily modified for objectives other than flattening the overall demand.

\section{References}

1. Melo, H., Heinrich, C.: Energy Balance in a Renewable Energy Community. IEEE EEEIC (May 2011)

2. Mulder, G., Ridder, F., Six, D.: Electricity Storage for Grid-connected Household Dwellings with PV Panels. Solar Energy 84, 1284-1293 (2010)

3. Cau, T., Kaye, R.: Multiple Distributed Energy Storage Scheduling Using Constructive Evolutionary Programming. In: Proc. of IEEE Power Engineering Society International Conference, vol. 22, pp. 402-407 (August 2002)

4. Vytelingum, P., Voice, T., Ramchurn, S., Rogers, A., Jennings, N.: Agent-based Micro-Storage Management for the Smart Grid. In: Proc. of 9th Int. Conf. on Autonomous Agents and Mutiagent Systems (AAMAS 2010) (May 2010)

5. Roozbehani, M., Dahleh, M., Mitter, S.: On the Stability of Wholesale Electricity Markets Under Real-Time Pricing. In: IEEE Conference on Decision and Control (CDC), pp. 1911-1918 (December 2010)

6. Kok, J., Warmer, C., Kamphuis, I.: PowerMatcher: Multi-Agent Control in Electicity Infrastructure. In: AAMAS 2005 (July 2005)

7. Ipakchi, A., Albuyeh, F.: Grid of The Future. IEEE Power and Energy Magazine, 52-62 (March 2009)

8. Houwing, M., Negenborn, R., Schutter, B.: Demand Response with Micro-CHP systems. Proceedings of the IEEE 99(1), 200-212 (2011)

9. Ahlert, K., Dinther, C.: Sensitivity Analysis of the Economic Benefits from Electricity Storage at the End Consumer Level. IEEE Transactions on PowerTech, 1-8 (October 2009) 